hep-th/9610147

OUTP-96-62P

October 1996

\title{
Two-dimensional dynamics of QCD3
}

\author{
B. Rusakov \\ Theoretical Physics, Oxford University \\ 1 Keble Road, Oxford, OX1 3NP, UK \\ e-mail: rusakov@thphys.ox.ac.uk
}

\begin{abstract}
.
Exact loop-variables formulation of pure gauge lattice $\mathrm{QCD}_{3}$ is derived from the Wilson version of the model. The observation is made that the resulting model is two-dimensional. This significant feature is shown to be a unique property of the gauge field. The model is defined on the infinite genus surface which covers regularly the original three-dimensional lattice. Similar transformation applied to the principal chiral field model in two and three dimensions for comparison with QCD.
\end{abstract}


QCD is believed to be a genuine model of the strong interaction. This belief is mainly based on the discovery of asymptotic freedom [1] and on the subsequent success of the perturbative description of ultra-violet physics. However, the main mystery of the strong interactions, its infra-red behavior and, in particular, the confinement of quarks, remains unexplained because of the lack of a non-perturbative solution. A number of 'mechanisms' of confinement have been suggested. However, no direct link has been unambiguously established between the model itself and any particular 'mechanism'. Nevertheless, the model is still analyzable by direct methods.

The unique, in many respects, non-perturbative formulation of QCD is the Wilson lattice model [2]. The partition function of the pure gauge model in $D$ dimensions has the form

$$
Z=\int \prod_{l} d U_{l} \prod_{p} d U_{p} \mathrm{e}^{\frac{N}{\lambda_{o}} \operatorname{tr}\left(U_{p}+U_{p}^{\dagger}\right)} \delta\left(U_{p}, \prod_{l \in p} U_{l}\right)
$$

where $\lambda_{o}$ is the bare coupling constant, $l$ and $p$ denote links and plaquettes of $D$-dimensional lattice, $U_{l}$ is the unitary matrix $(U(N)$ or $S U(N))$ attached to $l$-th link. In all the products the link variables are ordered.

The gauge-invariant $\delta$-function is

$$
\delta\left(U_{p}, \prod_{l} U_{l}\right)=\sum_{r} \chi_{r}\left(U_{p}^{\dagger}\right) \chi_{r}\left(\prod_{l} U_{l}\right)
$$

where $r$ is an irreducible representation with character $\chi_{r}(U)$.

The only non-zero observables in this model are invariant ordered products of link variables along closed loops, such as

$$
W(C)=\left\langle\frac{1}{N} \operatorname{tr} \prod_{l \in C} U_{l}\right\rangle
$$

( $C$ is the closed contour). It has been understood that the loop variables are relevant to solution of the quark confinement problem [2, 3]. In particular, as argued in [2], the simplest criterion of confinement is the area-law behavior of (3): $W(C) \sim \exp (-\sigma A)$, where $A$ is the area of the 'minimal' surface bounded by $C$ and $\sigma$ is the positive parameter (string tension) to be computed. On a lattice level, $A$ is the number of plaquettes of a disk bounded by $C$. Since the loop variables are only non-zero observables, it is clear that the model can be formulated in terms of these variables only. There is a number of works devoted to the loop dynamics (see review [4] and references therein). However, this has not led to a realization of confinement. In contrast, one can argue that if the model is a free theory in terms of the plaquette variables, then the area law is straightforward. Such realization requires direct reformulation of the partition function (1) to the loop (plaquette) variables. The latter is the main purpose of present paper円.

To rewrite partition function (11) in terms of the plaquette variables only, we have to perform integration over all link variables.

First, we replace (2) by

$$
\delta\left(I, U_{p}^{\dagger} \prod_{l} U_{l}\right)=\sum_{r} d_{r} \chi_{r}\left(U_{p}^{\dagger} \prod_{l} U_{l}\right),
$$

\footnotetext{
${ }^{1}$ See note added to the end of this paper.
} 


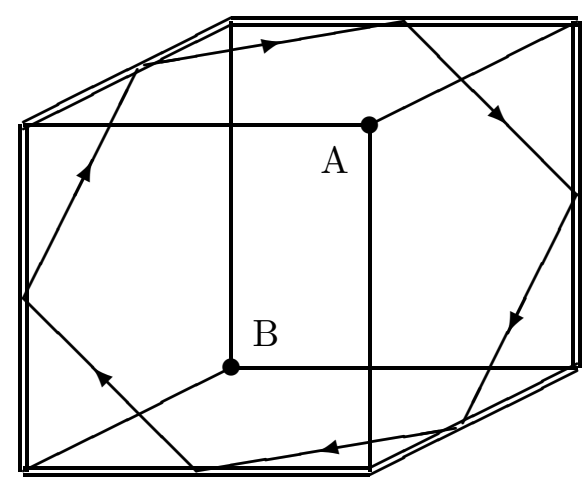

Figure 1: Choice of $A$ - and $B$-vertices and the hexagonal section of a cube.

where $d_{r}=\chi_{r}(I)$ is the dimension of representation. Such replacement does not change (1) due to invariance of the action with respect to similarity transformations $U_{p} \rightarrow \Omega U_{p} \Omega^{\dagger}$ and due to the formula

$$
\int d \Omega \chi_{r}\left(\Omega A \Omega^{\dagger} B\right)=\frac{1}{d_{r}} \chi_{r}(A) \chi_{r}(B) .
$$

However, there is a considerable difference in applications. In the case of definition (2), the $\Omega$-integrals are completely decoupled, while in the case of (\$), they play an important role. The reason we choose the definition (团), though at a first glance it seems an unnecessary complication, will soon become clear. Actually, we will use the freedom to choose the point of insertion of $U_{p}^{\dagger}$ into the product of $U_{l}$ 's in (荬), which means that we mark the point where the product around the plaquette starts (and ends).

Now we change the order of integration in (11) and consider the (topological) integral over link variables

$$
\int \prod_{l}^{\mathcal{N}_{1}-\mathcal{N}_{0}} d U_{l} \prod_{p}^{\mathcal{N}_{2}} \delta\left(U_{p}, \prod_{l \in p} U_{l}\right),
$$

where $\mathcal{N}_{k}$ is the number of $k$-simplexes: $\mathcal{N}_{0}$ is the number of vertices, $\mathcal{N}_{1}$ is the number of links etc. The number of independent integrals is $\mathcal{N}_{1}-\mathcal{N}_{0}$ due to the gauge invariance.

In this paper, we only consider the $3 \mathrm{~d}_{\text {case }}^{2}$. Due to the Euler theorem for the regular infinite 3d lattice,

$$
\mathcal{N}_{0}-\mathcal{N}_{1}+\mathcal{N}_{2}-\mathcal{N}_{3}=0
$$

the integral (6) must be proportional to the product of $\mathcal{N}_{3} \delta$-functions of $U_{p}$ 's.

Thus we have to find the set of $\mathcal{N}_{3}$ constraints (one for each cube) self-consistent with the original definition of the model. Let us choose two vertices, $A$ and $B$, of any cube, that are maximally remote from each other (Fig.1]), and imply the choice is valid for the neighboring cubes, i.e. the $A$-vertex is the same for all four cubes it belongs to, and similarly for $B$. Then, for the rest of the lattice, all $A$ - and $B$-vertices are uniquely defined. The boundary of any plaquette passes through either an $A$ - or a $B$-vertex and we call the corresponding plaquette either a $p_{A^{-}}$or a $p_{B}$-plaquette. Now we use our freedom in placing the $U_{p}$-matrices into the product around the plaquette 5 so that the product of links around the $p_{A}$-plaquettes starts

\footnotetext{
${ }^{2}$ The $2 \mathrm{~d}$ model is exactly solvable [⿰] considered in the next paper [7].

${ }^{3}$ See comments to (伍).
} 


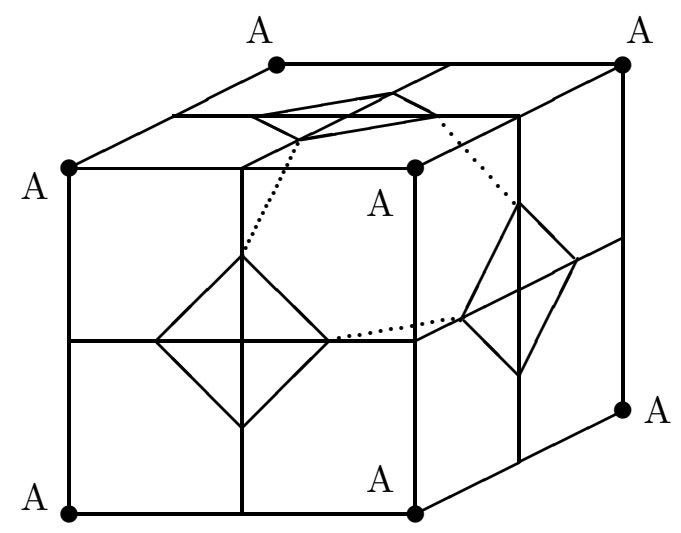

(a)

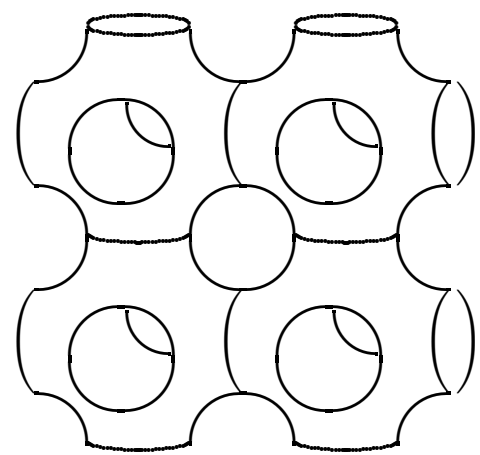

(b)

Figure 2: (a) The 8-cube's fragment of the lattice. A-vertices are the same as in Fig.1. Hexagons (sections of cubes) form the $2 \mathrm{~d}$ lattice (not all the lines are shown in order to not disturb the view). (b) The fragment of the resulting (smoothed) surface (corresponds to 32 cubes of original lattice).

at the vertex $A$, and similarly for $B$. Then, the invariant ordered product of three $U_{p_{A}}$ 's of each cube is equal to one of $U_{p_{B}}$ 's of the same cube, i.e.,

$$
\chi_{r}\left(\prod_{p_{A}} U_{p_{A}}\right)=\chi_{r}\left(\prod_{p_{B}} U_{p_{B}}\right)
$$

and both are equal to the invariant ordered product of $U_{l}$ 's, where l's form the closed loop separating $A$ and $B$ on the cube surface (double line in Fig.11). Then, the sought-for constraint is expressed by the formula:

$$
\delta\left(\prod_{p_{A}} U_{p_{A}}, \prod_{p_{B}} U_{p_{B}}\right)=\sum_{r} \chi_{r}\left(\prod_{p_{A}} U_{p_{A}}^{\dagger}\right) \chi_{r}\left(\prod_{p_{B}} U_{p_{B}}\right),
$$

where the $p_{A^{-}}$and $p_{B}$-plaquettes belong to the same cube. It is clear that all the constraints are independent. Thus, the integral (6) is equal (up to a possible constant factor) to the product of $\delta$-functions (8) taken over all cubes $c$. This has to be substituted into (1):

$$
Z=\int \prod_{p} d U_{p} \mathrm{e}^{\frac{N}{\lambda_{o}} \operatorname{tr}\left(U_{p}+U_{p}^{\dagger}\right)} \prod_{c} \delta\left(\prod_{p_{A} \in c} U_{p_{A}}, \prod_{p_{B} \in c} U_{p_{B}}\right)
$$

Thus, we formulated the original model (11) in terms of the plaquette variables only.

We notice now that this formulation has an interesting feature. Namely, (9) is the partition function of a certain two-dimensional model. To see this, let us consider the section of a cube by hexagon, as shown at Fig.1. Using the one-to-one correspondence between the plaquettes of original lattice and the links of the hexagon, we replace the $U_{p}$-variables in (9) by (new) $U_{l}$ 's where $l$ is the corresponding link of the hexagon.

The hexagons form the 2d lattice (Fig.2). The partition function (9), in terms of this lattice, takes the form

$$
Z=\int \prod_{l} d U_{l} \mathrm{e}^{\frac{N}{\lambda_{o}} \operatorname{tr}\left(U_{l}+U_{l}^{\dagger}\right)} \prod_{h} \delta\left(\prod_{l_{1} \in h} U_{l_{1}}, \prod_{l_{2} \in h} U_{l_{2}}\right),
$$


where $l$ and $h$ denote links and hexagons of the $2 \mathrm{~d}$ lattice. The order of links in any hexagon (argument of $\delta$-function) is $l_{1} l_{2} l_{1} l_{2} l_{1} l_{2}$ (at Fig.2(a), the $l_{2}$-type links correspond to the dotted lines). The fragment of the resulting $2 \mathrm{~d}$ lattice is shown at Fig.2(b). The whole lattice is easy to imagine - it is obtained from the regular 3d lattice (the lattice spacing is doubled with respect to the original $3 \mathrm{~d}$ lattice) by replacing links by the tubes (handles) and vertices by the smooth connections of tubes. The surface separates all $A$-vertices from all $B$-vertices. The two sets, being interior and exterior to the surface, are identical to each other. The genus $g$ of the surface is proportional to $\mathcal{N}_{3}$, namely, $g=\frac{3}{8} \mathcal{N}_{3}$.

Thus, we started with the three-dimensional field and discovered that, actually, interaction takes place on a certain surface. In our view, the picture becomes more clear in terms of the $r$ field (where $r$ is the $N$-component highest weight vector of representation; see definition (2)). Each particular sum over $r$ is attached to the particular hexagon and we can consider (10) as the partition function of statistical model of the $N$-component 2 d field $r$ ((10) is the sum over all $r$-configurations).

It is clear from the above that the reduction described is a unique property of the gauge field and takes place due to the gauge invariance of the original model (11). However, it is still interesting to see what happens in other models if similar change of variables is performed.

We consider the principal chiral field (PCF) model. Its partition function has the form

$$
Z_{\mathrm{PCF}}=\int \prod_{i} d U_{i} \prod_{l} d U_{l} \mathrm{e}^{\frac{N}{\lambda_{o}} \operatorname{tr}\left(U_{l}+U_{l}^{\dagger}\right)} \delta\left(U_{l=\{i j\}}, U_{i} U_{j}^{\dagger}\right)
$$

where $i, j$ are the vertex labels, $l$ denote links and $l=\{i j\}$ denotes the link between two neighboring vertices $i$ and $j$.

Now our aim is to formulate this model in terms of link variables only, i.e., to perform integration over vertices. Due to the Euler theorem, $\mathcal{N}_{0}-\mathcal{N}_{1}+\mathcal{N}_{2}=0$, the topological integral over vertices produces $\mathcal{N}_{2} \delta$-functions (or $\mathcal{N}_{2}-\mathcal{N}_{3}+\ldots \delta$-functions, if dimension is higher than two). The corresponding condition for the plaquette is, obviously,

$$
\delta\left(I, \prod_{l \in p} U_{l}\right)
$$

(this condition is valid for any loop, but we still keep the notation $p$ ). Thus,

$$
Z_{\mathrm{PCF}}=\int \prod_{l} d U_{l} \mathrm{e}^{\frac{N}{\lambda_{o}} \operatorname{tr}\left(U_{l}+U_{l}^{\dagger}\right)} \prod_{p} \delta\left(I, \prod_{l \in p} U_{l}\right) .
$$

Here, $p$ counts all independent loops, where 'independence' of the loop means an independence of the corresponding constraint. In particular, in the $2 \mathrm{~d}$ PCF all the loops are independent (unless this $2 \mathrm{~d}$ is the compact surface, in which case only one loop has to be excluded).

Let us consider PCF on the surface shown at Fig.2, in order to compare with $\mathrm{QCD}_{3}$. There are conditions $\delta\left(I, \prod_{l} U_{l}\right)$ at the non-contractible loops (the non-trivial cycles). Field $r$ corresponding to such a loop is attached to the section of the tube, i.e., there is a threedimensional interaction inside the handles, in sharp contrast to QCD.

If in (13) we replace the $\delta$-function by the heat kernel

$$
f(\alpha, U)=\sum_{r} d_{r} \mathrm{e}^{-\frac{\alpha}{N} C_{2}(r)} \chi_{r}(U)
$$


$\left(C_{2}(r)\right.$ is the second Casimir eigenvalue) and introduce a different couplings, $\alpha_{\text {hex }}$ for the hexagons, and $\alpha_{t u b}$ for the sections of the tubes, then $Z_{\mathrm{PCF}}$ is restored as the $\alpha_{\text {hex }}, \alpha_{t u b} \rightarrow 0$ limit of the result, while $\mathrm{QCD}_{3}$ partition function (10) is the $\alpha_{\text {hex }} \rightarrow 0, \alpha_{t u b} \rightarrow \infty$ limit.

There are several comments in order. The first one is the technical comment on use of (14) and (5) instead of (2). It has been understood already in $\mathrm{QCD}_{2}$ [6] that the $\Omega$-degrees of freedom, though they are hidden in the model, play an important role. In $\mathrm{QCD}_{2}$, they act by means of the formula (5) which pulls the powers of $d_{r}$ to denominator and, thus, the $1 / N$-expansion of the $\mathrm{QCD}_{2}$ partition function [6] can be done [8]. Here, in three dimensions, the use of the $\Omega$-degrees of freedom allowed us to decrease the effective dimension of the system. For reader familiar with the hermitian matrix models we remind that the similar situation takes place there. In all these cases, the use of the $\Omega$-variables has led to the kind of a 'stringy' description. It is worth to remind that it is these variables, which are precisely absent in any abelian model.

The important question is what happens with this picture in continuum limit. The loop variables are only non-zero observables in continuum theory as well. Therefore, the principal possibility to formulate the continuum model completely in terms of the loop variables still exists [3]. From the present consideration we see that the change of variables from links to plaquettes can be done at any lattice scale. Suppose we approach the continuum limit by refinement of the $3 \mathrm{~d}$ lattice so that the model is restored at each finer level. This should be achieved by the appropriate tuning of the coupling constant. Then at each level we can restore the described $2 \mathrm{~d}$ picture with the coupling constant corresponding to the current level of refinement. However, the $2 \mathrm{~d}$ lattice undergoes the infinite change of genus under refinement of the original $3 \mathrm{~d}$ lattice. The quantity which survives the refinement is the ratio $\mathcal{N}_{3} / g=8 / 3$, where $\mathcal{N}_{3}$ is the volume of the original lattice.

Note added: After submission of this work, I was informed of the related, pioneering work 9] on field strength and plaquette variable formulation of gauge theory. The relation between this early work and present paper is currently under investigation.

\section{References}

[1] D.Gross and F.Wilczek, Phys. Rev. D8 (1973) 3633 \& Phys. Rev. D9 (1974) 980.

[2] K.Wilson, Phys. Rev. D10 (1974) 2445.

[3] A.Polyakov, Gauge Fields and Strings, Harwood, 1987.

[4] A.Migdal, Phys. Rep. 102 (1983) 199.

[5] A.Migdal, ZhETF 69 (1975) 810.

[6] B.Rusakov, Mod. Phys. Lett. A5 (1990) 693.

[7] B.Rusakov, in preparation.

[8] D.Gross, Nucl. Phys. B400 (1993) 161; D.Gross and W.Taylor, Nucl. Phys. B400 (1993) 181 \& Nucl. Phys. B403 (1993) 395.

[9] M.B.Halpern, Phys. Rev. D19 (1979) 517; G.G.Batrouni, Nucl. Phys. B208 (1982) 12; 467; G.G.Batrouni and M.B.Halpern, Phys. Rev. D30 (1984) 1775; 1782. 